\title{
Geometric Framework for Unified Field Theory Using Finsler Gauge Transformation
}

\author{
Mallikarjuna Yallappa Kumbar, ${ }^{1}$ Sachin Jangir, ${ }^{1}$ \\ Chowdari Kondasandra Chowdappa, ${ }^{1}$ and Narasimhamurthy Senajji Kampalappa ${ }^{2}$ \\ ${ }^{1}$ Department of Mathematics, Vijaya College, Rastriya Vidyalaya Road, Bengaluru, Karnataka 560 004, India \\ ${ }^{2}$ Department of Mathematics, Kuvempu University, Shankarghatta, Karnataka 577 451, India
}

Correspondence should be addressed to Mallikarjuna Yallappa Kumbar; mallikarjunykumbar@gmail.com

Received 29 April 2016; Revised 14 July 2016; Accepted 23 July 2016

Academic Editor: Zisheng Wang

Copyright (C) 2016 Mallikarjuna Yallappa Kumbar et al. This is an open access article distributed under the Creative Commons Attribution License, which permits unrestricted use, distribution, and reproduction in any medium, provided the original work is properly cited.

We study the different types of Finsler space with $(\alpha, \beta)$-metrics which have nonholonomic frames as an application for classical mechanics and dynamics in physics using gauge transformation which helps to derive unified field theory. Further, we set up the application of Finsler geometry to geometrize the electromagnetic field completely.

\section{Introduction}

The common Finsler idea used by the physicists Beil and Holland is the existence of a nonholonomic frame on the vertical subbundle VTM of the tangent bundle of a base manifold $M$. This nonholonomic frame relates a semi-Riemannian metric (the Minkowski or the Lorentz metric) with an induced Finsler metric. In 2001, Antonelli and Bucataru have determined such a nonholonomic frame for two important classes of Finsler spaces that are dual in the sense of Randers and Kropina spaces [1, 2]. Recently, Bucataru and Miron have studied Finsler-Lagrange geometry and its applications to dynamical systems [3]. In this paper, the fundamental tensor field might be thought of as the result of two Finsler deformations. Then we can determine a corresponding frame for each of these two Finsler deformations. Consequently, a nonholonomic Finsler frame for a Finsler space with infinite series of $(\alpha, \beta)$-metric, that is, $F=\beta^{2} /(\beta-\alpha)$, will appear as a product of two Finsler frames formerly determined. We study the Finsler space with $(\alpha, \beta)$-metrics which have nonholonomic frames as an application for classical mechanics and dynamics in physics using gauge transformation which helps to derive unified field theory.

\section{Preliminaries}

We denote the tangent space at $x \in M$ by $T_{x} M$ and the tangent bundle of $M$ by TM. Each element of TM has the form $(x, y)$, where $x \in M$ and $y \in T_{x} M$. The natural projection $\pi: T M \rightarrow M$ is given by $\pi(x, y) \equiv x$.

A Finsler structure of $M$ is a function $F: T M \rightarrow[0, \infty)$, with the following properties:

(a) Regularity: $F$ is $C^{\infty}$ on the entire slit tangent bundle $T M \backslash\{0\}$

(b) Positive homogeneity: $F(x, \lambda y)=\lambda F(x, y)$ for all $\lambda>$ 0 .

(c) Strong convexity: $n \times n$ Hessian matrix $g_{i j}=$ $\left((1 / 2) F^{2}\right)_{y^{i} y^{j}}$ is positive definite at every point of $T M \backslash$ $\{0\}$, where we have used the notation ()$_{y}^{i}=\left(\partial / \partial y^{i}\right)()$, and the symbol TM\\{0\} means the tangent vector $y$ is nonzero in the tangent bundle TM.

Finsler geometry has its genesis in the integral of the form

$$
\int_{s}^{r} F\left(x^{1}, \ldots, x^{n} ; \frac{d x^{1}}{d t}, \ldots, \frac{d x^{n}}{d t}\right) d t .
$$


Throughout the project, the lowering and raising of indices are carried out by the fundamental tensor $g_{i j}$ defined above and its inverse matric tensor $g^{i j}$. It is obvious that the Finsler structure $F$ is a function of $\left(x^{i}, y^{i}\right)$. In this case, $F$ depends on $x^{i}$ only; then Finsler manifold reduces to a Riemannian manifold. The symmetric Cartan tensor can be defined as

$$
A_{i j k} \equiv \frac{F \partial g_{i j}}{2 \partial y^{k}}=\frac{F\left(F^{2}\right)_{y^{i} y^{j} y^{k}}}{4} .
$$

The Cartan tensor vanishes if and only if $g_{i j}$ has no $y$ dependence. So the Cartan tensor is a measurement of the deviation from the Riemannian manifold.

Using Euler's theorem on homogeneous function, we can get useful property of the fundamental tensor $g_{i j}$ and Cartan tensor $A_{i j k}$ :

$$
\begin{aligned}
g_{i j} l^{i} & =F_{y^{j}}, \\
g_{i j} l^{i} l^{j} & =1, \\
\frac{y^{i} \partial g_{i j}}{\partial y^{k}} & =0, \\
\frac{y^{j} \partial g_{i j}}{\partial y^{k}} & =0, \\
\frac{y^{k} \partial g_{i j}}{\partial y^{k}} & =0, \\
y^{i} A_{i j k} & :=y^{j} A_{i j k}:=y^{k} A_{i j k}=0,
\end{aligned}
$$

where $l^{i}=y^{i} / F$.

Definition 1. The Finsler space $F^{n}=\left(M^{n}, L\right)$ is said to have an $(\alpha, \beta)$-metric if $L$ is positively homogeneous function of degree one in two variables $\alpha=\sqrt{a_{i j}(x) y^{i} y^{j}}$ and $\beta=b_{i}(x) y^{i}$, where $\alpha$ is a Riemannian metric and $\beta$ is differential 1 -form.

An $(\alpha, \beta)$-metric is expressed in the following form:

$$
L=\alpha \phi(s), \quad s=\frac{\beta}{\alpha},
$$

where $\phi=\phi(s)$ is a positive function of $C^{\infty}$-class on an open interval $\left(-b_{0}, b_{0}\right)$. The norm $\left\|\beta_{x}\right\|_{\alpha}$ of $\beta$ with respect to $\alpha$ is defined by

$$
\begin{aligned}
\|\beta\|_{\alpha} & =\sup _{y \in T_{x} M}\{\beta(x, y), \alpha(x, y)\} \\
& =\sqrt{a^{i j}(x) b_{i}(x) b_{j}(x)} .
\end{aligned}
$$

In order to define $L, \beta$ must satisfy the condition $\left\|\beta_{x}\right\|_{\alpha}<$ $b_{0}$ for all $x \in M$. Thus the normalized element of support $l_{i}=\dot{\partial}_{i} L$ is given by

$$
l_{i}=\alpha^{-1} L_{\alpha} y_{i}+L_{\beta} b_{i}
$$

where

$$
y_{i}=a_{i j} y^{j} .
$$

The angular metric tensor $h_{i j}=L \dot{\partial}_{i} \dot{\partial}_{j} L$ is given by

$$
h_{i j}=p a_{i j}+q_{0} b_{i} b_{j}+q_{1}\left(b_{i} y_{j}+b_{j} y_{i}\right)+q_{2} y_{i} y_{j},
$$

where

$$
\begin{aligned}
& p=L L_{\alpha} \alpha^{-1}, \\
& q_{0}=L L_{\beta \beta}, \\
& q_{1}=L L_{\alpha \beta} \alpha^{-1}, \\
& q_{2}=\alpha^{-2} L\left(L_{\alpha \alpha}-L_{\alpha} \alpha^{-1}\right) .
\end{aligned}
$$

The fundamental tensor $g_{i j}=(1 / 2) \dot{\partial}_{i} \dot{\partial}_{j} L^{2}$ is given by

$$
g_{i j}=p a_{i j}+p_{0} b_{i} b_{j}+p_{1}\left(b_{i} y_{j}+b_{j} y_{i}\right)+p_{2} y_{i} y_{j},
$$

where

$$
\begin{aligned}
& p_{0}=q_{0}+L_{\beta}^{2}, \\
& p_{1}=q_{1}+L^{-1} p L_{\beta}, \\
& p_{2}=q_{2}+p^{2} L^{-2} .
\end{aligned}
$$

Moreover, the reciprocal tensor $g^{i j}$ of $g_{i j}$ is given by

$$
g^{i j}=p^{-1} a^{i j}+S_{0} b^{i} b^{j}+S_{1}\left(b^{i} y^{j}+b^{j} y^{i}\right)+S_{2} y^{i} y^{j},
$$

where

$$
\begin{aligned}
& S_{0}=\frac{\left(p p_{0}+\left(p_{0} p_{2}-p_{1}^{2}\right) \alpha^{2}\right)}{\zeta}, \\
& S_{1}=\frac{\left(p p_{1}+\left(p_{0} p_{2}-p_{1}^{2}\right) \beta\right)}{p \zeta}, \\
& S_{2}=\frac{\left(p p_{2}+\left(p_{0} p_{2}-p_{1}^{2}\right) b^{2}\right)}{p \zeta}, \\
& \zeta=p\left(p+p_{0} b^{2}+p_{1} \beta\right)+\left(p_{0} p_{2}-p_{1}^{2}\right)\left(\alpha^{2} b^{2}-\beta^{2}\right), \\
& b^{i}=a^{i j} b_{j}, \\
& b^{2}=a_{i j} b^{i} b^{j} .
\end{aligned}
$$

The $h \nu$-torsion tensor $C_{i j k}=(1 / 2) \dot{\partial}_{k} g_{i j}$ is given by

$$
2 p C_{i j k}=p_{1}\left(h_{i j} m_{k}+h_{j k} m_{i}+h_{k i} m_{j}\right)+v_{1} m_{i} m_{j} m_{k},
$$

where $v_{1}=p\left(\partial P_{0} / \partial \beta\right)-3 p_{1} q_{0} ; m_{i}=b_{i}-\alpha^{-2} \beta y_{i}$. 


\section{Nonholonomic Frames for Beil Metric}

We start with a real $n$-dimensional manifold $M$ of $C^{\infty}$ class. Denote by $(T M, \pi, M)$ the tangent bundle of the base manifold $M$ and by $(\widetilde{T M}, \pi, M)$ the tangent bundle with the null cross section removed. Local coordinates on $M$ are denoted by $\left(x^{i}\right)$, while the induced local coordinates on TM are denoted by $\left(x^{i}, y^{i}\right)$.

Denote by $\pi_{\star}$ the linear map induced by the canonical submersion $\pi: T M \rightarrow M$. As for every $u \in T M$, $\pi_{\star, u}: T_{u} T M \rightarrow T_{\pi(u)} M$ is an epimorphism; then its kernel determines $n$-dimensional distribution $V: u \in T M \mapsto$ $V_{u} T M=\operatorname{Ker} \pi_{\star, u} \subset T_{u} T M$. We call it the vertical distribution of the tangent bundle. If the natural basis of $T_{u} T M$ is denoted by $\left\{\left.\left(\partial / \partial x^{i}\right)\right|_{u},\left.\left(\partial / \partial y^{i}\right)\right|_{u}\right\}$, then $\left\{\left.\left(\partial / \partial y^{i}\right)\right|_{u}\right\}$ is a basis of $V_{u} T M$.

Definition 2. A Generalized Lagrange metric (GL-metric) is a metric $g$ on the vertical subbundle VTM of the tangent space $T M$; that is, for every $u \in T M, g_{u}: V_{u} T M \times V_{u} T M \rightarrow \mathbb{R}$ is bilinear, symmetric, of rank $n$, and of constant signature. A pair $\mathrm{GL}^{n}=(M, g)$ with GL-metric $g$ is called Generalized Lagrange space (GL-space).

In local coordinates, we denote $g_{i j}(u)=$ $g_{u}\left(\left.\left(\partial / \partial y^{i}\right)\right|_{u},\left.\left(\partial / \partial y^{j}\right)\right|_{u}\right)$ for every $u \in T M$. Then a GLmetric may be given by a collection of functions $g_{i j}(x, y)$ such that we have the following:

$\left(1^{0}\right) \operatorname{rank}\left(g_{i j}\right)=n ; g_{i j}(x, y)=g_{j i}(x, y)$.

$\left(2^{0}\right)$ The quadratic form $g_{i j}(x, y) \xi^{i} \xi^{j}$ has constant signature on $T M$.

$\left(3^{0}\right)$ If with respect to another system of local coordinates $\left(\tilde{x}^{i}, \tilde{y}^{i}\right)$ at $u \in T M$ we have $\tilde{g}_{k l}(x, y)=$ $g_{u}\left(\left.\left(\partial / \partial \widetilde{y}^{k}\right)\right|_{u},\left.\left(\partial / \partial \tilde{y}^{l}\right)\right|_{u}\right)$, then $g_{i j}$ and $\tilde{g}_{k l}$ are related by

$$
g_{i j}=\frac{\partial \widetilde{x}^{k}}{\partial x^{i}} \frac{\partial \widetilde{x}^{l}}{\partial x^{j}} \widetilde{g}_{k l} .
$$

Example 3. Consider $a_{i j}(x)$ are the components of a Riemannian metric on the base manifold $M$, consider $a^{\star}(x, y)>$ 0 and $b^{\star}(x, y) \geq 0$ are two Finsler scalars, and consider $B_{i}(x, y) d x^{i}$ is a Finsler 1 -form on TM. Then

$$
\begin{aligned}
g_{i j}(x, y)= & a^{\star}(x, y) a_{i j}(x) \\
& +b^{\star}(x, y) B_{i}(x, y) B_{j}(x, y)
\end{aligned}
$$

is a GL-metric [4], called the Beil metric. We say also that the metric tensor $g_{i j}$ is a Finsler deformation of the Riemannian metric $a_{i j}$. It has been studied and applied by R. Miron and R. K. Tavakol in General Relativity for $a^{\star}(x, y)=\exp (2 \sigma(x, y))$ and $b^{\star}=0$. The case $a^{\star}(x, y)=1$ with various choices of $b^{\star}$ and $B_{i}$ was introduced and studied by Beil for constructing a new unified field theory in [5]. Throughout this paper, we shall rise and lower indices only with the Riemannian metric $a_{i j}(x)$, that is, $y_{i}=a_{i j} y^{j}, b_{i}=a^{i j} b_{j}$, and so on. Let $U$ be an open set of TM and let

$$
V_{i}: u \in U \longmapsto V_{i}(u) \in V_{u} T M, \quad i \in\{1, \ldots, n\}
$$

be a vertical frame over $U$. If $V_{i}(u)=\left.V_{i}^{j}(u)\left(\partial / \partial y_{j}\right)\right|_{u}$, then $V_{i}^{j}(u)$ are the entries of a invertible matrix for all $u \in U$; denote by $\widetilde{V}_{k}^{j}(u)$ the inverse of this matrix. This means that

$$
\begin{aligned}
& V_{j}^{i} \widetilde{V}_{k}^{j}=\delta_{k}^{i}, \\
& \widetilde{V}_{j}^{i} V_{k}^{j}=\delta_{k}^{i} ;
\end{aligned}
$$

we call $V_{j}^{i}$ a nonholonomic Finsler frame.

Theorem 4. Consider a GL-space with Beil metric (16) and denote $B^{2}(x, y)=a_{i j}(x) B^{i}(x, y) B^{j}(x, y)$. Then

$$
V_{j}^{i}=\sqrt{a^{\star}} \delta_{j}^{i}-\frac{1}{B^{2}}\left(\sqrt{a^{\star}} \pm \sqrt{a^{\star}+b^{\star} B^{2}}\right) B^{i} B_{j}
$$

is a nonholonomic Finsler frame. The Beil metric (16) and the Riemannian metric $a_{i j}(x)$ are related by

$$
g_{i j}(x, y)=V_{i}^{k} V_{j}^{l}(x, y) a_{k l}(x) .
$$

Proof. Consider also

$$
\widetilde{V}_{k}^{j}=\frac{1}{\sqrt{a^{\star}}} \delta_{k}^{j}-\frac{1}{B^{2}}\left(\frac{1}{\sqrt{a^{\star}}} \pm \frac{1}{\sqrt{a^{\star}+b^{\star} B^{2}}}\right) B^{j} B_{k} .
$$

It is a direct calculation to check that $\widetilde{V}_{k}^{j}$ is the inverse of $V_{j}^{i}$; that is, $V_{j}^{i}$ is a nonholonomic frame. Next, we have that $V_{i}^{k} V_{j}^{l} a_{k l}=a^{\star} a_{i j}+b^{\star} B_{i} B_{j}=g_{i j}$, so the formula (21) holds true.

Remark 5. If we take $a^{\star}(x, y)=1$ and $b^{\star}(x, y)=k$, the nonholonomic Finsler frame (19) is the frame used by Beil in [6].

\subsection{Nonholonomic Frames for Finsler Spaces with $(\alpha, \beta)$-Metrics}

Definition 6. A Finsler space $F^{n}=(M, F(x, y))$ is called with $(\alpha, \beta)$-metric if there exists a 2-homogeneous function $L$ of two variables such that the Finsler metric $F: T M \rightarrow \mathbb{R}$ is given by

$$
F^{2}(x, y)=L^{\star}(\alpha(x, y), \beta(x, y)),
$$

where $\alpha(x, y)=\sqrt{a_{i j}(x) y^{i} y^{j}}$ is a Riemannian metric and $\beta(x, y)=b_{i}(x) y^{i}$ is a 1 -form on $M$.

Example 7. $\left(1^{0}\right)$ If $L^{\star}(\alpha, \beta)=(\alpha+\beta)^{2}$, then the Finsler space with Finsler metric $F(x, y)=\left(a_{i j} y^{i} y^{j}\right)^{1 / 2}+b_{i}(x) y^{i}$ is called a Randers space.

$\left(2^{0}\right)$ If $L^{\star}(\alpha, \beta)=\alpha^{4} / \beta^{2}$, then the Finsler space with Finsler metric $F(x, y)=a_{i j}(x) y^{i} y^{j} /\left|b_{i}(x) y^{i}\right|$ is called a Kropina space.

These classes of Finsler spaces play an important role in Finsler geometry and they are dual in the sense of Hrimiuc and Shimada [7]. 
For a Finsler space with $(\alpha, \beta)$-metric $F^{2}(x, y)=$ $L^{\star}(\alpha(x, y), \beta(x, y))$, we have the Finsler invariants [8]:

$$
\begin{aligned}
\rho & =\frac{1}{2 \alpha} \frac{\partial L^{\star}}{\partial \alpha} ; \\
\rho_{0} & =\frac{1}{2} \frac{\partial^{2} L^{\star}}{\partial \beta^{2}} ; \\
\rho_{-1} & =\frac{1}{2 \alpha} \frac{\partial^{2} L^{\star}}{\partial \alpha \partial \beta} ; \\
\rho_{-2} & =\frac{1}{2 \alpha^{2}}\left(\frac{\partial^{2} L^{\star}}{\partial \alpha^{2}}-\frac{1}{\alpha} \frac{\partial L^{\star}}{\partial \alpha}\right) .
\end{aligned}
$$

For a Finsler space with $(\alpha, \beta)$-metric we have

$$
\rho_{-1} \beta+\rho_{-2} \alpha^{2}=0 \text {. }
$$

With respect to these notations, we have that the metric tensor $g_{i j}$ of a Finsler space with $(\alpha, \beta)$-metric is given by [8]

$$
\begin{aligned}
g_{i j}(x, y)= & \rho a_{i j}(x)+\rho_{0} b_{i}(x) b_{j}(x) \\
& +\rho_{-1}\left(b_{i}(x) y_{j}+b_{j}(x) y_{i}\right)+\rho_{-2} y_{i} y_{j} .
\end{aligned}
$$

The metric tensor $g_{i j}$ of a Lagrange space with $(\alpha, \beta)$-metric can be arranged into the following form:

$$
\begin{aligned}
g_{i j}= & \rho a_{i j}+\frac{1}{\rho_{-2}}\left(\rho_{-1} b_{i}+\rho_{-2} y_{i}\right)\left(\rho_{-1} b_{j}+\rho_{-2} y_{j}\right) \\
& +\frac{1}{\rho_{-2}}\left(\rho_{0} \rho_{-2}-\rho_{-1}^{2}\right) b_{i} b_{j} .
\end{aligned}
$$

From (26) we can see that $g_{i j}$ is the result of two Finsler deformations:

$$
\begin{aligned}
& a_{i j} \longmapsto h_{i j} \\
& h_{i j}=\rho a_{i j}+\frac{1}{\rho_{-2}}\left(\rho_{-1} b_{i}+\rho_{-2} y_{i}\right)\left(\rho_{-1} b_{j}+\rho_{-2} y_{j}\right) \\
& h_{i j} \longmapsto g_{i j} \\
& g_{i j}=h_{i j}+\frac{1}{\rho_{-2}}\left(\rho_{0} \rho_{-2}-\rho_{-1}^{2}\right) b_{i} b_{j} .
\end{aligned}
$$

The nonholonomic Finsler frame that corresponds to the first deformation of (27) is, according to Theorem 4, given by

$$
\begin{gathered}
V_{j}^{i}=\sqrt{\rho} \delta_{j}^{i}-\frac{1}{B^{2}}\left(\sqrt{\rho} \pm \sqrt{\rho+\frac{B^{2}}{\rho_{-2}}}\right)\left(\rho_{-1} b^{i}+\rho_{-2} y^{i}\right) \\
\cdot\left(\rho_{-1} b_{j}+\rho_{-2} y_{j}\right)
\end{gathered}
$$

where $B^{2}=a_{i j}\left(\rho_{-1} b^{i}+\rho_{-2} y^{i}\right)\left(\rho_{-1} b^{j}+\rho_{-2} y^{j}\right)=\rho_{-1}^{2} b^{2}+\beta \rho_{-1} \rho_{-2}$. The metric tensors $a_{i j}$ and $h_{i j}$ are related by

$$
h_{i j}=V_{i}^{k} V_{j}^{l} a_{k l} \text {. }
$$

According to Theorem 4, the nonholonomic Finsler frame that corresponds to the second deformation of (27) is given by

$$
\widetilde{V}_{j}^{i}=\delta_{j}^{i}-\frac{1}{C^{2}}\left(1 \pm \sqrt{1+\frac{\rho_{-2} C^{2}}{\rho_{0} \rho_{-2}-\rho_{-1}^{2}}}\right) b^{i} b_{j}
$$

where $C^{2}=h_{i j} b^{i} b^{j}=\rho b^{2}+\left(1 / \rho_{-2}\right)\left(\rho_{-1} b^{2}+\rho_{-2} \beta\right)^{2}$. The metric tensors $h_{i j}$ and $g_{i j}$ are related by the following formula:

$$
g_{m n}=\widetilde{V}_{m}^{i} \widetilde{V}_{n}^{j} h_{i j}
$$

From (29) and (31), we have that $X_{m}^{k}=V_{i}^{k} \widetilde{V}_{m}^{i}$ with $V_{i}^{k}$ given by (28) and $\widetilde{V}_{m}^{i}$ given by (30) is a nonholonomic Finsler frame of the Finsler space with $(\alpha, \beta)$-metric.

3.2. Nonholonomic Frame for Infinite Series of $(\alpha, \beta)$-Metric. Now we will consider particular Finsler $(\alpha, \beta)$-metric; that is, $L^{\star}=F^{2}=\left(\beta^{2} /(\beta-\alpha)\right)^{2}$; by (23), we have the Finsler invariants:

$$
\begin{aligned}
\rho= & \left(\frac{\beta^{4}}{\alpha(\beta-\alpha)^{3}}\right), \\
\rho_{0}= & \left(\frac{4 \beta^{5}+2 \beta^{4} \alpha-6 \beta^{2} \alpha^{3}-3 \beta^{4}-4 \beta^{3} \alpha}{(\beta-\alpha)^{4}}\right), \\
\rho_{-1}= & \left(\frac{\beta^{4}-4 \alpha \beta^{3}}{\alpha(\beta-\alpha)^{4}}\right), \\
\rho_{-2}= & \left(\frac{8 \alpha \beta^{4}-2 \beta^{5}}{2 \alpha^{3}(\beta-\alpha)^{4}}\right), \\
B^{2}= & {\left[\frac{\left(\beta^{4}-4 \alpha \beta^{3}\right)^{2}}{\alpha^{2}(\beta-\alpha)^{8}}\right] b^{2} } \\
& +\frac{1}{(\beta-\alpha)^{4}}\left[\frac{8 \beta^{9}}{\alpha^{3}}-\frac{\beta^{10}}{\alpha^{4}}-\frac{16 \beta^{8}}{\alpha^{2}}\right] .
\end{aligned}
$$

The nonholonomic Finsler frame that corresponds to the first deformation of (27) is, according to Theorem 4 , given by

$$
\begin{aligned}
V_{j}^{i} & =\left\{\frac{\beta^{4}}{\alpha(\beta-\alpha)^{3}}\right\}^{1 / 2} \delta_{j}^{i}-\frac{1}{B^{2}}\left[\left\{\frac{\beta^{4}}{\alpha(\beta-\alpha)^{3}}\right\}^{1 / 2}\right. \\
& \pm\left\{\left(\frac{\beta^{4}}{\alpha(\beta-\alpha)^{3}}\right)\right. \\
& \left.\left.+\frac{B^{2}}{\left(\left(8 \alpha \beta^{4}-2 \beta^{5}\right) / 2 \alpha^{3}(\beta-\alpha)^{4}\right)}\right\}^{1 / 2}\right] \\
& \cdot\left(\left(\frac{\beta^{4}-4 \alpha \beta^{3}}{\alpha(\beta-\alpha)^{4}}\right) b^{i}+\left(\frac{8 \alpha \beta^{4}-2 \beta^{5}}{2 \alpha^{3}(\beta-\alpha)^{4}}\right) y^{i}\right) \\
& \cdot\left(\left(\frac{\beta^{4}-4 \alpha \beta^{3}}{\alpha(\beta-\alpha)^{4}}\right) b_{j}+\left(\frac{8 \alpha \beta^{4}-2 \beta^{5}}{2 \alpha^{3}(\beta-\alpha)^{4}}\right) y_{j}\right) .
\end{aligned}
$$


According to Theorem 4, the nonholonomic Finsler frame that corresponds to the second deformation of (27) is given by

$$
\begin{aligned}
\widetilde{V}_{j}^{i} & =\delta_{j}^{i}-\frac{1}{C^{2}}(1 \pm\{1 \\
& \left.\left.+\frac{C^{2}\left(\left(8 \alpha \beta^{4}-2 \beta^{5}\right) / 2 \alpha^{3}(\beta-\alpha)^{4}\right)}{\left(\left(4 \beta^{5}+2 \beta^{4} \alpha-6 \beta^{2} \alpha^{3}-3 \beta^{4}-4 \beta^{3} \alpha\right) /(\beta-\alpha)^{4}\right)\left(\left(8 \alpha \beta^{4}-2 \beta^{5}\right) / 2 \alpha^{3}(\beta-\alpha)^{4}\right)-\left(\left(\beta^{4}-4 \alpha \beta^{3}\right) / \alpha(\beta-\alpha)^{4}\right)^{2}}\right)^{1 / 2}\right) \\
& \cdot b^{i} b_{j},
\end{aligned}
$$

where

$$
\begin{aligned}
C^{2} & =\left(\frac{\beta^{4}}{\alpha(\beta-\alpha)^{3}}\right) b^{2} \\
& +\frac{1}{\left(\left(8 \alpha \beta^{4}-2 \beta^{5}\right) / 2 \alpha^{3}(\beta-\alpha)^{4}\right)}\left(\left(\frac{\beta^{4}-4 \alpha \beta^{3}}{\alpha(\beta-\alpha)^{4}}\right)\right. \\
& \left.\cdot b^{2}+\left(\frac{8 \alpha \beta^{4}-2 \beta^{5}}{2 \alpha^{3}(\beta-\alpha)^{4}}\right) \beta\right)^{2} .
\end{aligned}
$$

Theorem 8. Consider a Finsler space $L^{\star}=F^{2}=\left(\beta^{2} /(\beta-\right.$ $\alpha))^{2}$, for which the condition (24) is true; then $X_{j}^{i}=V_{k}^{i} \widetilde{V}_{j}^{k}$ is a Finslerian nonholonomic frame with $V_{k}^{i}$ and $\widetilde{V}_{j}^{k}$ being given by (33) and (34), respectively.

\section{Finsler Gauge Transformation}

If a particle in a space time moves along a curved, nongeodesic path, then it is said that the particle is under the influence of some external force. In such a case, an external force term is added to the equation of motions to explain the path of motion. Alternative point of view is that motion can be explained by a new metric, which would result from a gauge transformation. In this way, physical force fields can be geometrized, and general relativistic idea of space time curvature determining the path of the particle will also include fields other than gravitation. For this purpose, a class of gauge transformations which act on tangent space is considered. There are actually several ways to introduce Finsler geometry. Probably the most common way is just to assume a certain form for the metric function $F$. It would be nice, however, to have a more physical picture of where the metric comes from. It is proposed to show that nontrivial Finsler metrics can be obtained from a certain type of Gauge transformation. This transformation takes a Lorentz space, of the sort we have been discussing, into another kind of space where the metrics and other geometrical quantities are dependent not only on $x$ but also on the tangent coordinate $y$.
The Gauge transformation is defined as follows:

$$
\bar{y}^{\mu}=\widetilde{V}_{v}^{\mu} y^{\nu}
$$

where $\widetilde{V}$ is a nonsingular matrix with inverse $V$ :

$$
\widetilde{V}_{\nu}^{\mu} V_{\lambda}^{\nu}=\delta_{\lambda}^{\mu}
$$

This transformation acts on coordinates $y$ of the fiber. The action on the vertical basis is

$$
\frac{\partial}{\partial \bar{y}^{\alpha}}=V_{\alpha}^{\gamma} \frac{\partial}{\partial y^{\gamma}} .
$$

This gives a new internal or fiber metric:

$$
h_{\alpha \beta}=\eta_{\gamma \delta} V_{\alpha}^{\gamma} V_{\beta}^{\delta}
$$

So the gauge transformation is a diffeomorphism acting on the vertical (fiber) subspace of $T M$. The $V$ matrices could be a representation of any subgroup of GL $(4 ; R)$. This transformation is sometimes called a pure gauge transformation. It is also comparable to the metric $G$ group of Beil [5]. It does not act directly on coordinates $x^{\mu}$ of the horizontal subspace of $T M$. That is, $\bar{x}=x$. Even so, it does produce a change of the base space metric. One way to infer the metric change is to require that the length of the tangent vector $y^{\mu}$ in the original Lorentz space,

$$
F^{2}=\eta_{\mu \nu} y^{\mu} y^{\nu}
$$

be invariant under the transformation. The expression $F^{2}$ is used here since this is, indeed, the form of the Finsler metric function in the Lorentz space. So the transformed metric function, the length of the new tangent vector, is

$$
\widetilde{F}^{2}=g_{\mu \nu} \bar{y}^{\mu} \bar{y}^{\nu}
$$

where $g_{\mu \nu}$ is the new base space metric. If the components of a vector are changed under a transformation, then the metric is changed to preserve invariance. One way to see how the metric of $M$ changes is to consider that, following the soldering,

$$
g_{\mu \nu}=h_{\alpha \beta} e_{\mu}^{\alpha} e_{\nu}^{\beta}
$$


$e_{\mu}^{\alpha}$ are just orthonormal tetrads. This actually produces what is called soldering of the two parts of TM. One result of this soldering is that the components $y^{\mu}$ of a certain vector in $M$ are identified with the coordinates $y^{\mu}$ of the vertical part of $T M$. In other words, the base metric is related to the fiber metric by the same Lorentz transformation. This can also be written in the form

$$
g_{\mu \nu}=\eta_{\gamma \delta} b_{\mu}^{\gamma} b_{\nu}^{\delta},
$$

where

$$
b_{\mu}^{\alpha}=V_{\beta}^{\alpha} e_{\mu}^{\beta}
$$

is a new tetrad which is not necessarily orthonormal. It is possible to construct unified theories in Finsler manifold using these tetrads for fiber coordinates. So the gauge transformation acting on the vertical part of TM gives not only a new fiber metric but also a new metric on the horizontal part of TM. This is a new metric on the base space $M$. It should be emphasized, though, that there is no $x$-coordinate transformation involved here. It is easy to see that this type of gauge transformation generates a way to get Finsler spaces. The transformation matrix $V$ just has to be not only a function of $x$ but also a function of the tangent coordinates $y$ :

$$
V_{v}^{\mu}=V_{v}^{\mu}\left(x^{\mu}, y^{\nu}\right)
$$

and the new metric $g$ is $y$-dependent:

$$
g_{\mu \nu}=V_{\mu}^{\sigma} V_{\nu}^{\rho} \eta_{\sigma \rho}
$$

As will be seen, the matrix $V$ can also include a general vector field which is not necessarily the tangent vector. This vector can be the gauge potential itself, or it can be related to the potential vector by a "gauge" phase transformation. Note that (46) results from the assumption that the orthonormal tetrad $e_{\mu}^{\alpha}$ is just $\delta_{\mu}^{\alpha}$. This is a matter of convenience without consequence to the main argument. The metric $g$ is not, in general, itself the Finsler metric. In order to get the Finsler metric, we take

$$
\widetilde{F}^{2}=g_{\mu \nu} y^{\mu} y^{\nu} .
$$

This should be compared with (41). The function (47) should be used since we will be concerned with the effect of the change of metric with respect to $y$ rather than $\bar{y}$. This is actually the canonical Finsler approach and is used by Bao et al. [9-11]. A new metric $f_{\mu \nu}$ is then computed in the standard way:

$$
f_{\mu \nu}=\frac{1}{2} \frac{\partial^{2} \widetilde{F}^{2}}{\partial y^{\mu} \partial y^{\nu}} .
$$

We say that this is a Finsler metric if

$$
\widetilde{F}^{2}=f_{\mu \nu} y^{\mu} y^{\nu} .
$$

As discussed above, we do not insist that the metric be positive definite. Another point of curiosity is the difference between the metrics $g$ and $f$. Of course, if $V$ is not $y$ dependent, we have $f=g$ and the new metric is only Riemannian. Actually, $f=g$ under more general conditions. One only needs the "metric condition" of Asanov [10]:

$$
y^{\nu} \frac{\partial g_{\mu \nu}}{\partial y^{\alpha}}=0
$$

It is of interest that (50) is satisfied by the Randers and Weyl metrics but not, in general, by the metric (46). For physical reasons we want $\widetilde{F}^{2}$ to be of second-degree homogeneity in $y$. For example, if $L$ is the Lagrangian, then the energy is

$$
E=\frac{\partial L^{\star}}{\partial y^{\mu}} y^{\mu}-L^{\star} .
$$

Ordinarily,

$$
L^{\star}=\widetilde{F}^{2},
$$

so if $L^{\star}$ is of second-degree homogeneity, then $E=L^{\star}$ which is good for mechanical systems. For another choice, with $L^{\star}$ of first-degree homogeneity, as is possible in the Lagrangian theories of Miron and Anastasiei [12], $E=0$ and there is a problem of how to explain a system with zero energy.

There is a way around this homogeneity problem [12], which involves an energy function, but it is simpler just to choose $\widetilde{F}^{2}$ to be of second-degree homogeneity. This also relates naturally to the original metric function $F^{2}=\eta_{\mu \nu} y^{\mu} y^{\nu}$. This means that the transformation matrix $V$ is of zero-degree homogeneity in $y$ :

$$
\frac{\partial V_{v}^{\mu}}{\partial y^{\alpha}} y^{\alpha}=0
$$

It is of interest to ask how many of the known Finsler metrics can be obtained by this sort of gauge transformation? At this point, one can only list those for which a specific $V$ matrix is known: Randers, Kropina, Beil, Weyl, and metrics where $V$ gives a conformal transformation. Obviously, nonlinear metrics are not included. What does this gauge transformation mean physically? It can be interpreted as what happens when a nongravitational field is turned on in a region of space. For example, the field could be electromagnetic. A metric has also been given for the electroweak field $S U(2) \times U(1)$ [13]. The gauge transformation could also be interpreted as a distortion or deformation of the original Lorentz space. In other words, the gauge field twists or distorts the space. The relative effect is, by the way, a torsion rather than a curvature. Although, remarkably, the final outcome is a curved space. The torsion interpretation has been advocated by Holland [14] who relates the transformation to nonholonomic frames. The nonholonomic frame viewpoint is explained in a very useful new paper by Bucataru [15]. There is a teleparallel relation between the original Lorentz space and the resulting Finsler space. The change in local connections between the two spaces is zero in suitable coordinates. This implies a generalized equivalence principle which will be discussed below. One can write, in the natural basis $\left(d x^{\mu}, d y^{\nu}\right)$,

$$
\omega_{\rho}^{\sigma}=d V_{\mu}^{\sigma} \widetilde{V}_{\rho}^{\mu}=L_{\rho k}^{\star \sigma} d x^{k}+B_{\rho \lambda}^{\sigma} d y^{\lambda},
$$


defining the horizontal $L^{\star}$ and vertical $B$ components of the connection. Given the metric condition (49), (48) reduces to

$$
\begin{aligned}
f_{\mu \nu}= & \eta_{\alpha \beta} V_{\mu}^{\alpha} V_{\nu}^{\beta}, \\
d f_{\mu \nu}= & \eta_{\alpha \beta}\left(d V_{\mu}^{\alpha} V_{\nu}^{\beta}+V_{\mu}^{\alpha} d V_{\nu}^{\beta}\right) \\
= & f_{\sigma \nu} L_{\mu k}^{\star \sigma} d x^{k}+f_{\sigma \nu} B_{\mu \lambda}^{\sigma} d y^{\lambda}+f_{\mu \rho} L_{\nu k}^{\star \rho} d x^{k} \\
& +f_{\mu \rho} B_{\nu \lambda}^{\rho} d y^{\lambda} .
\end{aligned}
$$

From another point of view,

$$
d f_{\mu \nu}=\frac{\partial f_{\mu \nu}}{\partial x^{k}} d x^{k}+\frac{\partial f_{\mu \nu}}{\partial y^{\lambda}} d y^{\lambda}
$$

A comparison of (56) and (57) gives

$$
\begin{aligned}
& \frac{\partial f_{\mu \nu}}{\partial x^{k}}=f_{\sigma \nu} L_{\mu k}^{\star \sigma}+f_{\mu \rho} L_{\nu k}^{\star \rho}=L_{\nu \mu k}^{\star}+L_{\mu \nu k}^{\star}, \\
& \frac{\partial f_{\mu \nu}}{\partial y^{\lambda}}=f_{\sigma \nu} B_{\mu \lambda}^{\sigma}+f_{\mu \rho} B_{\nu \lambda}^{\rho}=B_{\nu \mu \lambda}+B_{\mu \nu \lambda} .
\end{aligned}
$$

These are just the horizontal and vertical components of $d f_{\mu \nu}$. $L^{\star}$ and $B$ have in general no index symmetry. The net result of the work so far is a nontrivial Finsler metric and a Finsler metric function. In other words, this is the point where most Finsler theories begin. So what is the use of all these preliminaries? The main benefit is a physical understanding of how a Finsler space might describe a space which contains a nongravitational field. That is, it has been shown how a gauge transformation takes a Lorentz space to a space which is Finslerian. Note that the inverse transformation takes a metric from a Finsler metric back to the Lorentz metric. This demonstrates a generalized equivalence, whereby a transformation exists, which produces a local inertial frame along the world line of a particle. This means that the motion of a particle along a curved path not only might be due to a gravitational field derived from a metric but also might be due to other metric produced fields. It will be shown shortly exactly how this occurs. First, though, some standard Finsler results are presented. A significant point is that these results are developed in terms of a coordinate transformation of the base space $M$. This contrasts with the gauge transformation just depicted which is a vertical diffeomorphism, a transformation in the fiber space.

The gauge transformation is used to get the Finsler space. The connections given so far describe the transition to that space. The coordinate transformation deals with the properties of the resulting Finsler space. It describes the translation (sometimes called the transplantation) as one moves from one point to another in the space. The coordinate basis of $M$ does not transform covariantly under a coordinate transformation on $T M$. One has to introduce the local adapted basis

$$
\frac{\delta}{\delta x^{\mu}}=\frac{\partial}{\partial x^{\mu}}-N_{\mu}^{\nu} \frac{\partial}{\partial y^{\nu}}
$$

where $N$ is the nonlinear connection. The adapted basis on $T M$ is

$$
\left(\frac{\delta}{\delta x^{\mu}}, \frac{\delta}{\delta y^{\mu}}\right)
$$

and the dual basis is

$$
\left(d x^{\mu}, \delta y^{\mu}=d y^{\mu}+N_{\nu}^{\mu} d x^{\nu}\right)
$$

which do transform covariantly under a coordinate transformation.

The behavior of the metric under the coordinate transformation is, in the adapted basis,

$$
d f_{\mu \nu}=\frac{\delta f_{\mu \nu}}{\delta x^{k}} d x^{k}+\frac{\delta f_{\mu \nu}}{\delta y^{\lambda}} \delta y^{\lambda}
$$

or it is, in the natural basis,

$$
d f_{\mu \nu}=\frac{\partial f_{\mu \nu}}{\partial x^{k}} d x^{k}+\frac{\partial f_{\mu \nu}}{\partial y^{\lambda}} \delta y^{\lambda}
$$

This leads immediately to the usual connections. Consider

$$
F_{\mu \nu k}=\frac{1}{2}\left(\frac{\delta f_{\mu \nu}}{\delta x^{k}}+\frac{\delta f_{k \mu}}{\delta x^{\nu}}-\frac{\delta f_{k \nu}}{\delta x^{\mu}}\right)
$$

is the adapted horizontal connection. This is symmetric in the second and third indices. Consider

$$
C_{\mu \lambda \nu}=\frac{1}{2}\left(\frac{\delta f_{\mu \nu}}{\delta y^{\lambda}}+\frac{\delta f_{\lambda \mu}}{\delta y^{\nu}}-\frac{\delta f_{\lambda \nu}}{\delta y^{\mu}}\right)=\frac{1}{2}\left(\frac{\delta f_{\mu \nu}}{\delta y^{\lambda}}\right)
$$

is the vertical connection. It is symmetric in all indices and by (58) is related to the vertical connection obtained from the gauge transformation by

$$
C_{\mu \nu \lambda}=\frac{1}{2}\left(B_{\mu \nu \lambda}+B_{\nu \mu \lambda}\right)
$$

One can also consider the Finsler-Christoffel connection

$$
\Gamma_{\mu \nu k}=\frac{1}{2}\left(\frac{\partial f_{\mu \nu}}{\partial x^{k}}+\frac{\partial f_{k \mu}}{\partial x^{\nu}}-\frac{\partial f_{k \nu}}{\partial x^{\mu}}\right) .
$$

One can then form the canonical Finsler connections, for example, the Cartan connection $(N, F, C)$. Also, the various Finsler torsions and curvatures can be obtained. A highly recommended source for this standard theory is the book by Miron and Anastasiei [12]. For the Riemannian case, it is well known that there is curvature but no torsion. There is an interesting result which can be obtained using (58):

$$
\Gamma_{\mu \nu k}=\frac{1}{2}\left(L_{\mu \nu k}+L_{\nu \mu k}+L_{k \mu \nu}+L_{\mu k \nu}-L_{k \nu \mu}-L_{v k \mu}\right) .
$$

This shows that the Finsler-Christoffel connection can be computed from the horizontal gauge connection. The FinslerChristoffel connection is probably the most interesting to physicists, since it appears in the geodesic equation, the equation of motion, $d \nu_{\lambda} / d \tau+\gamma_{\lambda \mu \nu} v^{\mu} v^{\nu}=0$. It is assumed 
that there is a time like path with parameter $y$ such that $v^{\alpha}=d x^{\alpha} / d \tau$ and $\eta_{\alpha \beta} v^{\alpha} v^{\beta}=c^{2}$ holds. It is noted in passing that the equation of motion can also be written as

$$
\frac{d}{d \tau}\left(\frac{\partial \widetilde{F}^{2}}{\partial v^{\mu}}\right)-\frac{\partial \widetilde{F}^{2}}{\partial x^{\mu}}=0,
$$

which is the usual Euler-Lagrange equation.

\section{Application}

It is now time to get some specific physics using the above developments. There are several gauge transformations which might give useful results. One of them is now examined and compared. They are given by $V_{\nu}^{\mu}$ and $\widetilde{V}_{\lambda}^{\nu}$ as (28) and (30), respectively.

It will be assumed that the vector $B^{\mu}$ is related to the electromagnetic potential vector $A^{\mu}$ by

$$
B^{\mu}=A^{\mu}+\frac{\partial \Lambda}{\partial x^{\nu}} \eta^{\mu \nu}
$$

This shows how the potential is included in a gauge transformation. Equation (70) has the form of what is commonly called a "gauge" transformation but which more properly should be called a phase transformation. Equation (28) corresponds to the actual mathematical diffeomorphism [12] which is a pure gauge transformation. Again, $A^{\mu}$ is not directly associated with the nonlinear connection in (30).

It is not difficult to show how a transformation like (28) is directly derived from $U(1)$ group [6]; also $B^{2}=\eta_{\mu \nu} B^{\mu} B^{\nu}$.

The potential $A_{\mu}$ is known to be given by

$$
A_{\mu}=-\frac{m c}{e} v_{\mu}+\frac{\partial \phi}{\partial x^{\mu}} .
$$

If $v^{\mu}$ is changed by (28), then the potential is also changed. For example, it can be transformed from zero to a nonzero vector. This means that the electromagnetic potential can be "turned on" by the transformation (28). The metric which is associated with this transformation is

$$
g_{\mu \nu}=\eta_{\sigma \rho} V_{\mu}^{\sigma} V_{\nu}^{\rho}=\eta_{\mu \nu}+\mathfrak{\Re} B_{\mu} B_{\nu}
$$

This has the form of a Kaluza-Klein metric except that the vector potential $B$ appears instead of $A$. Also, the space $M$ is four-dimensional, not five-dimensional. Furthermore, in Kaluza-Klein theories, the vector $B$ is a special case of a connection. Here, $B$ is specially not associated with a connection. It will be seen that the field $F_{\mu \nu}$ which is derived from $B$ is a part of the connection.

In general, $B$ is a function of both $x$ and $y$. There is a variety of possible Finsler geometries. Metrics of this type have been labeled "Beil" metrics $[4,15,16]$. The metric function is

$$
\widetilde{F}^{2}=\left(\frac{\beta^{2}}{\beta-\alpha}\right)^{2}
$$

where $\alpha=\left(\eta_{\mu \nu} v^{\mu} v^{\nu}\right)^{1 / 2}, \beta=\boldsymbol{\Omega}^{1 / 2} B_{\mu} v^{\mu}$, and $\boldsymbol{\Omega}$ is a constant which turns out to be just a factor times the universal gravitational constant. In order to illustrate the usefulness of this metric, we take the simplest case, which is the case where $B$ is a function of $x$ only. This means that the resulting Riemann space is actually the osculating space to this class of Finsler spaces.

The transformation connection is easy to derive:

$$
\begin{aligned}
L_{\lambda \mu \nu}= & B^{-2}\left[1-\left(1+\Re B^{2}\right)^{1 / 2}\right]\left[B_{\lambda} \frac{\partial B_{\mu}}{\partial x^{\nu}}-\frac{\partial B_{\lambda}}{\partial x^{\nu}} B_{\mu}\right] \\
& +\Re B_{\lambda} \frac{\partial B_{\mu}}{\partial x^{\nu}}
\end{aligned}
$$

and, from (68),

$$
\begin{aligned}
& \Gamma_{\lambda \mu \nu} \\
& =\frac{1}{2} k\left[B_{\lambda}\left(\frac{\partial B_{\mu}}{\partial x^{\nu}}+\frac{\partial B_{v}}{\partial x^{\mu}}\right)\right] \\
& +\frac{1}{2} k\left[B_{\mu}\left(\frac{\partial B_{\lambda}}{\partial x^{\nu}}-\frac{\partial B_{v}}{\partial x^{\lambda}}\right)+B_{\nu}\left(\frac{\partial B_{\lambda}}{\partial x^{\mu}}-\frac{\partial B_{\mu}}{\partial x^{\lambda}}\right)\right] \text {. }
\end{aligned}
$$

The condition

$$
B_{\nu} v^{\nu}=\frac{e}{m c k}
$$

is imposed, which implies

$$
\frac{\partial B_{v}}{\partial x^{\mu}} v^{\nu}=\frac{\partial}{\partial x^{\mu}}\left(B_{v} v^{\nu}\right)=0
$$

In [17], Beil has proved this. The geodesic equation becomes

$$
\frac{d v_{\lambda}}{d \tau}+\frac{e}{m c}\left(\frac{\partial B_{\lambda}}{\partial x^{\mu}}-\frac{\partial B_{\mu}}{\partial x^{\lambda}}\right) v^{\mu}=0
$$

One can identify

$$
F_{\mu \lambda}=\frac{\partial B_{\lambda}}{\partial x^{\mu}}-\frac{\partial B_{\mu}}{\partial x^{\lambda}}
$$

and (78) is the Lorentz equation of motion for a charged particle, recalling (70). Note that condition (76) does not restrict the gauge freedom of the electromagnetic potential $A_{\mu}$. The field $F_{\mu \nu}$ can be identified as the electromagnetic field.

This means that a purely geometric derivation of electromagnetism has been developed. $U(1)$ symmetry determines the gauge transformation which in turn produces the metric and the rest of the structure.

In the present theory, all potentials are included in a metric which transforms like a metric. All fields are included in a connection which transforms like a connection. The equations of motion are geodesic equations. The energymomentum for all fields is derived from a curvature. 
By way of comparison, consider three other gauge transformations which produce Finsler metrics which can be related to the one just studied. One transformation is

$$
\begin{aligned}
V_{j}^{i} & =\left\{\frac{\beta^{4}}{\alpha(\beta-\alpha)^{3}}\right\}^{1 / 2} \delta_{j}^{i}-\frac{1}{B^{2}}\left[\left\{\frac{\beta^{4}}{\alpha(\beta-\alpha)^{3}}\right\}^{1 / 2} \pm\left\{\left(\frac{\beta^{4}}{\alpha(\beta-\alpha)^{3}}\right)+\frac{B^{2}}{\left(\left(8 \alpha \beta^{4}-2 \beta^{5}\right) / 2 \alpha^{3}(\beta-\alpha)^{4}\right)}\right\}^{1 / 2}\right] \\
& \cdot\left(\left(\frac{\beta^{4}-4 \alpha \beta^{3}}{\alpha(\beta-\alpha)^{4}}\right) b^{i}+\left(\frac{8 \alpha \beta^{4}-2 \beta^{5}}{2 \alpha^{3}(\beta-\alpha)^{4}}\right) y^{i}\right)\left(\left(\frac{\beta^{4}-4 \alpha \beta^{3}}{\alpha(\beta-\alpha)^{4}}\right) b_{j}+\left(\frac{8 \alpha \beta^{4}-2 \beta^{5}}{2 \alpha^{3}(\beta-\alpha)^{4}}\right) y_{j}\right), \\
\widetilde{V}_{j}^{i} & =\delta_{j}^{i}-\frac{1}{C^{2}}(1 \pm\{1 \\
& \left.\left.+\frac{C^{2}\left(\left(8 \alpha \beta^{4}-2 \beta^{5}\right) / 2 \alpha^{3}(\beta-\alpha)^{4}\right)}{\left(\left(4 \beta^{5}+2 \beta^{4} \alpha-6 \beta^{2} \alpha^{3}-3 \beta^{4}-4 \beta^{3} \alpha\right) /(\beta-\alpha)^{4}\right)\left(\left(8 \alpha \beta^{4}-2 \beta^{5}\right) / 2 \alpha^{3}(\beta-\alpha)^{4}\right)-\left(\left(\beta^{4}-4 \alpha \beta^{3}\right) / \alpha(\beta-\alpha)^{4}\right)^{2}}\right\}^{1 / 2}\right) \\
& \cdot b^{i} b_{j} .
\end{aligned}
$$

The resulting metric $g$ is (46). The metric $f_{\mu \nu}$ computed according to (48) is identical to (46), which approaches Finsler metric function:

$$
F^{2}=\left(\frac{\beta^{2}}{\beta-\alpha}\right)^{2}
$$

this is the special Finsler $(\alpha, \beta)$-metric function. The connections for this symmetric are well known and also complicated; hence they will not be repeated here. This is to be compared with Lorentz equation (see [18, eq (3)]) for charged particles to the equation of motion of a charged particle as geodesic equation (78), where one can see that $\beta$ is undetermined.

\section{Conclusion}

In 1982, Holland studied a unified formalism that uses nonholonomic frames on space time arising from consideration of a charged particle moving in an external electromagnetic field $[19,20]$. In 1987, Ingarden was first to point out that the Lorentz force law can be written in this case as geodesic equation on a Finsler space called Randers space [21]. In 1995, Beil viewed a gauge transformation as nonholonomic frame on the tangent bundle of a four-dimensional manifold $[12,22]$. The geometry that follows from these considerations gives a unified approach to gravitation and gauge symmetries. Considering the above concepts, we have presented a geometric setup that allows us to obtain necessary and sufficient conditions for the existence of invariants for certain types of nonholonomic systems for Finsler $(\alpha, \beta)$-metrics. Our methods have been successfully applied to prove the existence and nonexistence of invariants for concrete problems. Moreover, our geometric framework generates a new setup that might be useful to determine conditions that generate the existence of invariants for systems with a particular class of $(\alpha, \beta)$-metrics that we plan to study concerning nonholonomic systems for which some interesting results concern the existence. Finally, in this paper, we set up the application of Finsler geometry to geometrize the electromagnetic field completely. First Finsler gauge transformations are considered; thus, by a specific transformation, Finsler metric function is calculated and properties of this metric function are studied. Finally, general forms of Finsler metric functions, resulting from this transformation, are considered.

\section{Competing Interests}

The authors declare that they have no competing interests.

\section{References}

[1] P. L. Antonelli and I. Bucataru, "Finsler connections in anholonomic geometry of a Kropina space," Nonlinear Studies, vol. 8, no. 1, pp. 171-184, 2001.

[2] P. L. Antonelli and I. Bucataru, "On Holland's frame for Randers space and its applications in physics," in Steps in Differential Geometry, Proceedings of the Colloquium on Differential Geometry, 25-30 July, 2000, Debrecen, Hungary, Institute of Mathematics and Informatics, 2001.

[3] I. Bucataru and R. Miron, "Finsler-Lagrange geometry, applications to dynamical systems," CEEXET 3174/2005-2007, CEEX M III 12595/2007, 2007.

[4] M. Anastasiei and H. Shimada, "Beil metrics associated to a Finsler space," Balkan Journal of Geometry and its Applications (BJGA), vol. 3, no. 2, pp. 1-16, 1998.

[5] R. G. Beil, "Finsler and kaluza-klein gauge theories," International Journal of Theoretical Physics, vol. 32, no. 6, pp. 1021-1031, 1993. 
[6] R. G. Beil, "Finsler gauge transformations and general relativity," International Journal of Theoretical Physics, vol. 31, no. 6, pp. 1025-1044, 1992.

[7] D. Hrimiuc and H. Shimada, "On the L-duality between Lagrange and Hamilton manifolds," Nonlinear World, vol. 3, no. 4, pp. 613-641, 1996.

[8] M. Matsumoto, Foundations of Finsler Geometry and Special Finsler Spaces, Kaisheisha Press, Otsu, Japan, 1986.

[9] D. Bao, S. S. Chern, and Z. Shen, An Introduction to RiemannFinsler Geometry, Springer, New York, NY, USA, 2000.

[10] G. S. Asanov, Finsler Geometry, Relativity and Gauge Theories, Reidel, Dordrecht, The Netherlands, 1985.

[11] P. L. Antonelli, R. S. Ingarden, and M. Matsumoto, The Theory of Sprays and Finsler Spaces with Applications in Physics and Biology, vol. 58 of Fundamental Theories of Physics, Kluwer Academic Publishers, Dordrecht, The Netherlands, 1993.

[12] R. Miron and M. Anastasiei, The Geometry of Lagrange Spaces: Theory and Applications, vol. 59 of Fundamental Theories of Physics, Kluwer Academic Publishers, Dordrecht, The Netherlands, 1994.

[13] R. G. Beil, "Electroweak symmetry on the tangent bundle," International Journal of Theoretical Physics, vol. 40, no. 2, pp. 591-601, 2001.

[14] P. R. Holland, "Geometry of dislocated de Broglie waves," Foundations of Physics, vol. 17, no. 4, pp. 345-363, 1987.

[15] I. Bucataru, "Nonholonomic frames in Finsler geometry," Balkan Journal of Geometry and Its Applications, vol. 7, no. 1, pp. 13-27, 2002.

[16] V. Balan, "Synge-Beil and Riemann-Jacobi jet structures with applications to physics," International Journal of Mathematics and Mathematical Sciences, vol. 2003, no. 27, pp. 1693-1702, 2003.

[17] R. G. Beil, "Equations of motion from Finsler geometric methods," in Finslerian Geometries: A Meeting of Minds, P. L. Antonelli, Ed., vol. 109 of Fundamental Theories of Physics, pp. 95-109, Kluwer Academic, Dordrecht, The Netherlands, 2000.

[18] R. G. Beil, "Finsler geometry and relativistic field theory," Foundations of Physics, vol. 33, no. 7, pp. 1107-1127, 2003.

[19] P. R. Holland and C. Philippidis, "Anholonomic deformations in the ether: a significance for the electrodynamic potentials," in Quantum Implications, B. J. Hiley and F. D. Peat, Eds., pp. 295-311, Routledge and Kegan Paul, New York, NY, USA, 1987.

[20] P. R. Holland, "Electromagnetism, particles and anholonomy," Physics Letters A, vol. 91, no. 6, pp. 275-278, 1982.

[21] R. S. Ingarden, "On physical interpretations of Finsler \& Kawagneli spaces," Tensor, vol. 46, pp. 354-360, 1987.

[22] R. G. Beil, "Electrodynamics from a metric," International Journal of Theoretical Physics, vol. 26, no. 2, pp. 189-197, 1987. 


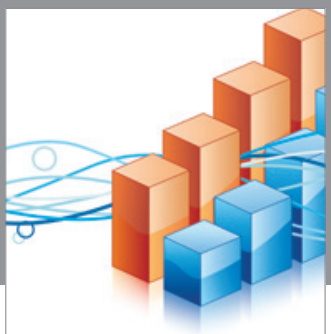

Advances in

Operations Research

vatem alat4

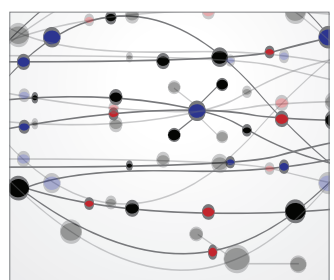

\section{The Scientific} World Journal
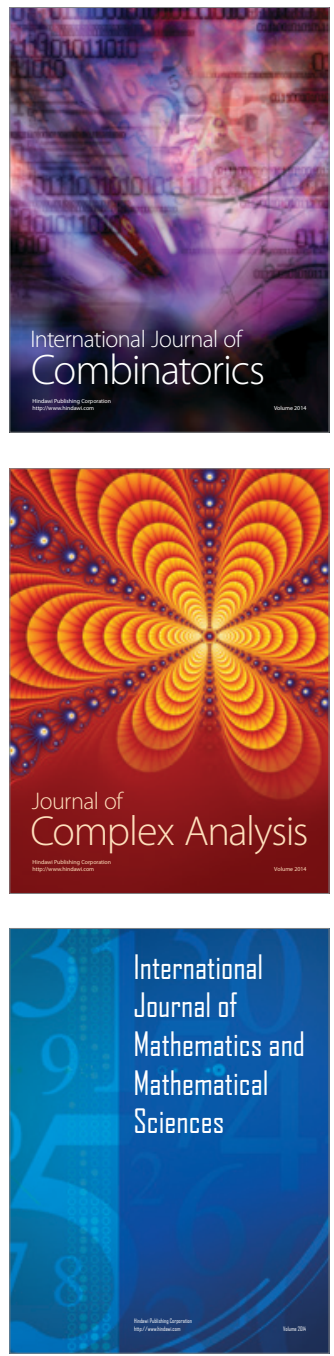
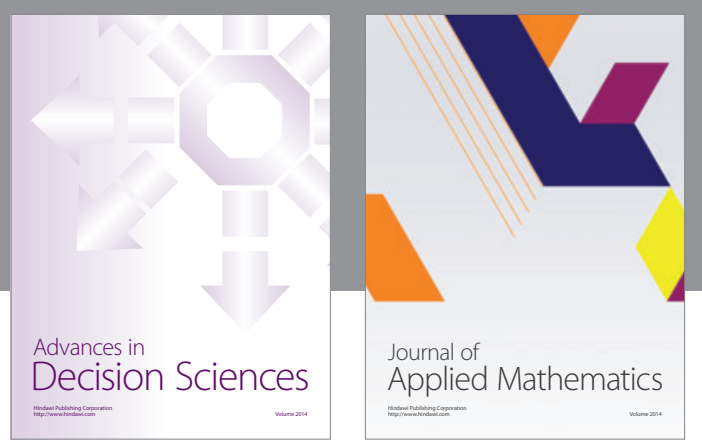

Algebra

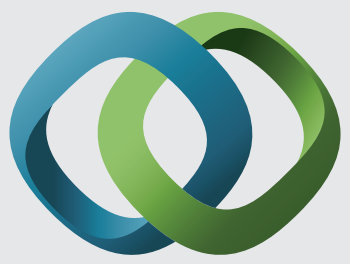

\section{Hindawi}

Submit your manuscripts at

http://www.hindawi.com
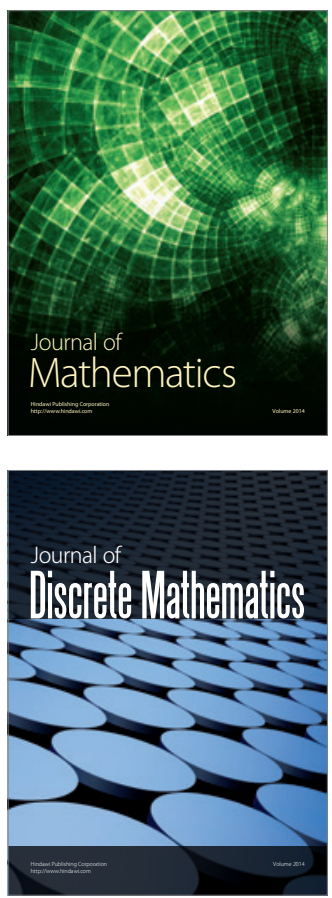

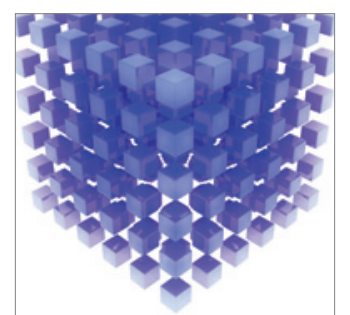

Mathematical Problems in Engineering
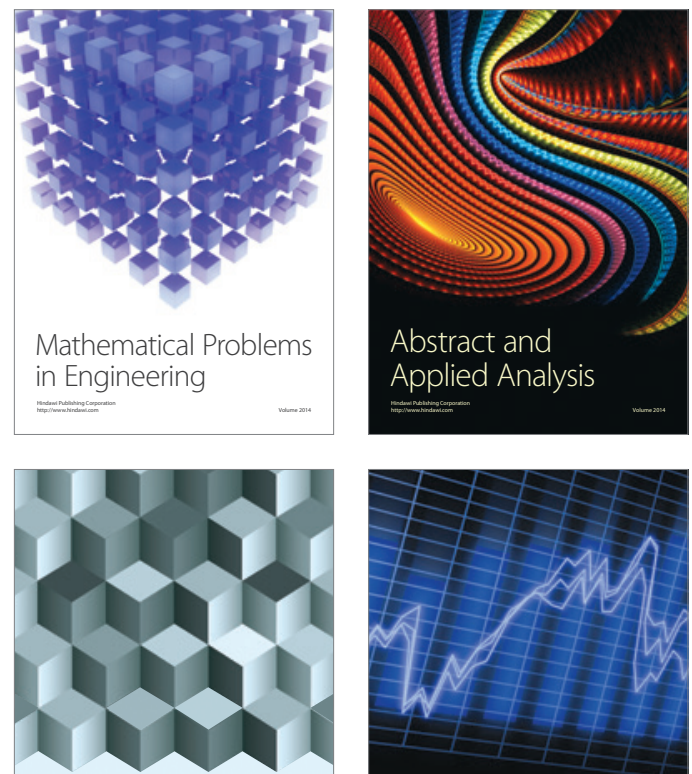

Journal of

Function Spaces

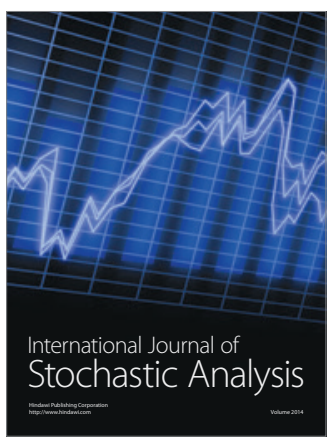

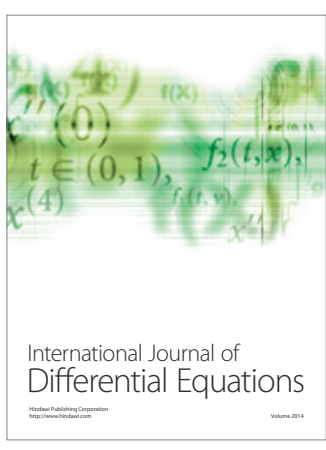
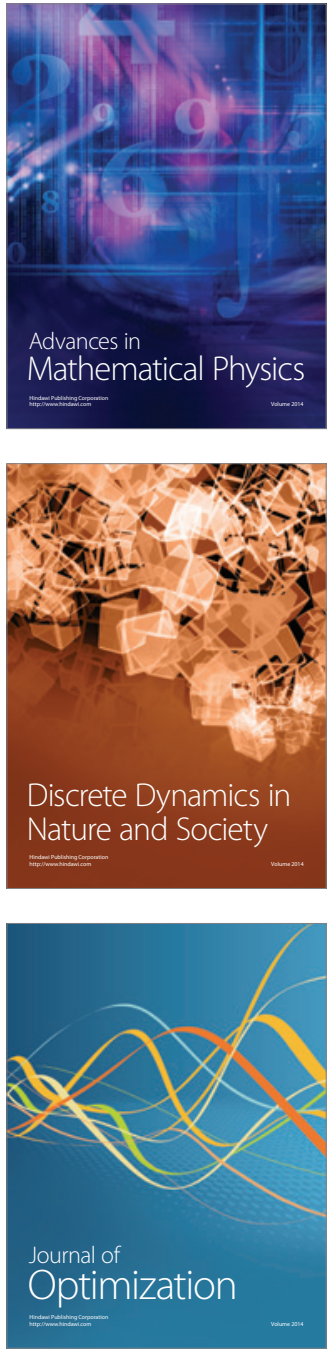